Pacific Journal of Mathematics

ON THE CONFORMAL MAPPING OF VARIABLE REGIONS 


\title{
ON THE CONFORMAL MAPPING OF VARIABLE REGIONS
}

\author{
Frank J. POLANSKY
}

We establish an estimate for the functional

$$
I(f, g ; \rho)=\int_{C_{\rho}}|f(t)-g(t)| \cdot|d t|,
$$

$C_{\rho}$ is the circumference $|t|=\rho, 0 \leqq \rho<1$. Here $f$ and $g$ are normalized conformal mapping functions of $|z|<1$ onto a pair of bounded, open, simply connected, origin containing domains in the $w$ plane whose boundaries are near each other in some sense. In the second part of the paper we establish an estimate for the functional $I\left(f^{\prime}, \mathbf{g}^{\prime} ; \rho\right)$ in case the boundaries are additionally assumed to be rectifiable.

We are motivated by the fact that if one of the domains is a disc we get the case of "nearly circular" domains which has been much studied.

Aside from an absolute constant our estimates are geometric in nature, being expressed in terms of numbers which are derived from properties of the boundaries of the mapped domains. They are of interest to us because they hold uniformly for all $\rho, 0 \leqq \rho<1$ and because they approach zero when one of the domains converges to the other as described in the paper.

1. Definition 1. Let $D_{f}$ and $D_{g}$ denote a pair of open, bounded, simply connected sets in the $w$ plane both of which contain the origin. Let $\Gamma_{f}$ and $\Gamma_{g}$ denote their respective boundaries. Let $\Delta$ denote the component of $D_{f} \cap D_{g}$ which contains the origin and let $\Gamma$ denote the boundary of $\Delta$. Let $\lambda_{f}$ be the radius of the largest disk lying in the complement of $\Gamma_{f}$ and having its center on $\Gamma$ (if no such disk exists, write $\lambda_{f}=0$ ). Let $\lambda_{g}$ be analogously defined. The inner distance is defined by the formula

$$
\varepsilon=\varepsilon\left(\Gamma_{f}, \Gamma_{g}\right)=\operatorname{Max}\left(\lambda_{f}, \lambda_{g}\right)
$$

The statement ' $\phi(z)$ is a normalized mapping function' means that $\phi(z)$ is the conformal mapping function of one bounded, simply connected, origin containing domain onto another and that $\phi(0)=0$, and $\phi^{\prime}(0)$ is positive.

The symbol $C_{\rho}$ will always be used to denote the locus $|t|=\rho$, $0 \leqq \rho<1$.

Let $R_{1}$ and $R_{2}$ denote the radii of two circles with centers at $w=0$ 
which are such that the boundaries $\Gamma_{f}$ and $\Gamma_{g}$ lie in the ring

$$
0<R_{1} \leqq|w| \leqq R_{2} \text {. }
$$

THEOREM 1. If $f(z)$ and $g(z)$ are the normalized mapping functions of $|z|<1$ onto $D_{f}$ and $D_{g}$ respectively, if $0<\varepsilon\left(\Gamma_{f}, \Gamma_{g}\right)<R_{1}$, then

$$
I(f, g ; \rho)=\int_{C_{\rho}}|f(t)-g(t)| \cdot|d t| \leqq K_{1} R_{2}\left(\frac{\varepsilon}{R_{1}}\right)^{1 / 6} .
$$

The number $K_{1}$ is an absolute constant, and the inequality holds uniformly for all $\rho, 0 \leqq \rho<1$.

Before proving Theorem 1 we state some results which are used in the proof.

Lemma A. ([4], p. 349.) Let $D$ be a bounded, simply connected domain which contains the origin and let $z=\psi(w)$ be the normalized mapping function of $D$ onto the disk $|z|<1$ in the $z$ plane. If $w$ is a point of $D$ at a distance $\delta$ from the boundary of $D$, then

$$
1-|\psi(w)| \leqq 4 \sqrt{\delta \psi^{\prime}(0)}
$$

LEMMA B. ([3], p. 563.) Let $w=\phi(z)$ be the normalized mapping function of $|z|<1$ onto the domain whose boundary $D$ lies in the ring $1-\sigma \leqq|w| \leqq 1,0<\sigma<1$. Then

$$
\int_{C_{\rho}}|\phi(t)-t|^{2} \cdot|d t| \leqq K_{2} \sigma^{2}
$$

The number $K_{2}$ is an absolute constant, and inequality holds uniformly for all $\rho, 0 \leqq \rho<1$.

LEMma C. ([1], p. 165.) If $F(z)$ and $\Theta(z)$ are regular in $|z|<1$ if $\Theta(0)=0$ and $|\Theta(z)|<1$ in $|z|<1$, then

$$
\int_{C_{\rho}}|F(\Theta(t))|^{2} \cdot|d t| \leqq \int_{C_{\rho}}|F(t)|^{2} \cdot|d t|,
$$

uniformly valid for all $\rho, 0 \leqq \rho<1$.

2. Proof of Theorem 1. (a) From Definition 1, each point of $\Gamma$ will have distance at most $\varepsilon$ from $\Gamma_{f}$. The inverse of $f(z)$ maps $\Delta$ onto a domain $E$ which lies in $|z|<1$. Let $E_{1}$ denote the boundary of $E$. From Lemma $A$, the set $E_{1}$ will lie in the ring

$$
1-4 \sqrt{\frac{\varepsilon}{f^{\prime}(0)}} \leqq|z| \leqq 1 \text {. }
$$


Since

$$
f^{\prime}(0) \geqq \inf _{|z|<1}\left|\frac{f(z)}{z}\right| \geqq R_{1},
$$

the set $E_{1}$ will lie in the ring

$$
1-4 \sqrt{\frac{\varepsilon}{R_{1}}} \leqq|z| \leqq 1
$$

The above inequality fails to define a ring if $\varepsilon / R_{1} \geqq 1 / 16$. We treat the two cases separately. Let $\omega(z)$ be the normalized mapping function of $|z|<1$ onto $E$. If $\varepsilon / R_{1}<1 / 16$, we have from Lemma B,

$$
J(\rho)=\int_{C_{\rho}}|\omega(t)-t|^{2}|d t| \leqq 16 K_{2} \frac{\varepsilon}{R_{1}} .
$$

For the case $1 / 16 \leqq \varepsilon / R_{1}<1$, we have trivially,

$$
J(\rho) \leqq 4 \cdot 2 \pi \rho \leqq 128 \pi \cdot \frac{\varepsilon}{R_{1}} .
$$

Thus, if $K_{3}=\operatorname{Max}\left[128 \pi, 16 K_{2}\right]$, then

$$
J(\rho) \leqq K_{3} \frac{\varepsilon}{R_{1}}, 0<\varepsilon<R_{1} .
$$

(b) For $0 \leqq r \leqq 1,|z|<1$ let

$$
B_{r}(z)=f(z)-f(r z) .
$$

Then

$$
f(z)-f(\omega(z))=B_{r}(z)-B_{r}(\omega(z))+f(r z)-f(r \omega(z)) .
$$

Hence

(2)

$$
\begin{aligned}
& \int_{C_{\rho}}|f(t)-f(\omega(t))| \cdot|d t| \\
& \leqq \\
& \quad \int_{C_{\rho}}\left|B_{r}(t)\right| \cdot|d t|+\int_{C_{\rho}}\left|B_{r}(\omega(t))\right| \cdot|d t| \\
& \quad+\int_{C_{\rho}}|f(r t)-f(r \omega(t))| \cdot|d t| \equiv I_{1}+I_{2}+I_{3} .
\end{aligned}
$$

If $f(z)=\sum_{1}^{\infty} a_{k} z^{k}$ then

$$
\begin{aligned}
I_{1}^{2} & \leqq 2 \pi \rho \cdot \int_{C_{\rho}}\left|B_{r}(t)\right|^{2} \cdot|d t|=2 \pi \rho \Sigma\left|a_{k}\right|^{2} \cdot \rho^{2 k} \cdot\left(1-r^{k}\right)^{2} \cdot 2 \pi \rho \\
& \leqq 4 \pi^{2} \Sigma\left|a_{k}\right|^{2}\left(1-r^{k}\right) \\
& =4 \pi^{2} \Sigma\left|a_{k}\right|^{2}(1-r)\left(1+r+r^{2}+\cdots+r^{k-1}\right)
\end{aligned}
$$




$$
\begin{aligned}
& \leqq 4 \pi^{2}(1-r) \Sigma\left(\left|a_{k}\right|^{2} \cdot k\right)=4 \pi(1-r) \cdot\left(\text { area of } D_{f}\right) \\
& \leqq 4 \pi(1-r) \cdot \pi R_{2}^{2} .
\end{aligned}
$$

Thus, if $K_{4}^{2}=4 \pi^{2}$,

$$
I_{1} \leqq K_{4} R_{2} \sqrt{1-r}, 0 \leqq r \leqq 1 .
$$

From Lemma $\mathrm{C}$, the same bound is valid for $I_{2}$ :

$$
I_{2} \leqq K_{4} R_{2} \sqrt{1-r}, 0 \leqq r \leqq 1 .
$$

(c) If $0<r<\alpha<1$, we have for the integrand of: $I_{3}$ :

$$
\begin{aligned}
|f(r t)-f(r \omega(t))| & \leqq \frac{1}{2 \pi} \int_{C_{\alpha}}|f(\gamma)| \cdot\left|\frac{1}{\gamma-r t}-\frac{1}{\gamma-r \omega}\right| \cdot|d \gamma| \\
& \leqq \frac{1}{2 \pi} \int_{C_{\alpha}}|f(\gamma)| \cdot\left|\frac{r \omega-r t}{(\gamma-r t)(\gamma-r \omega)}\right| \cdot|d \gamma| \\
& \leqq \frac{\sup |f| \cdot r|\omega-t|}{2 \pi} \int_{C_{\alpha}} \frac{|d \gamma|}{|\gamma-r t| \cdot|\gamma-r \omega|} \\
& \leqq \frac{R_{2}|\omega-t|}{2 \pi}\left[\int_{C_{\alpha}} \frac{|d \gamma|}{|\gamma-r t|^{2}}\right]^{1 / 2} \cdot\left[\int_{C_{\alpha}} \frac{|d \gamma|}{|\gamma-r \omega|^{2}}\right]^{1 / 2} \\
& \leqq \frac{R_{2}|\omega-t|}{2 \pi}\left[\frac{2 \pi \alpha}{\alpha^{2}-|r t|^{2}}\right]^{1 / 2} \cdot\left[\frac{2 \pi \alpha}{\alpha^{2}-|r \omega|^{2}}\right]^{1 / 2} .
\end{aligned}
$$

Let $\alpha \rightarrow 1$ and we obtain

$$
|f(r t)-f(r \omega(t))| \leqq \frac{R_{2}|\omega(t)-t|}{1-r}, 0<r<1 .
$$

Hence, from (1)

$$
\begin{aligned}
\int_{C_{\rho}}|f(r t)-f(r \omega(t))| \cdot|d t| & \leqq \frac{R_{2}}{1-r} \int_{C_{\rho}}|\omega(t)-t||d t| \\
& \leqq \frac{R_{2}}{1-r}\left[\int_{C_{\rho}}|\omega-t|^{2}|d t|\right]^{1 / 2} \cdot \sqrt{2 \pi \rho} \\
& \leqq \frac{R_{2}}{1-r}\left[2 \pi K_{3}\left(\frac{\varepsilon \varepsilon}{R_{1}}\right)\right]^{1 / 2} .
\end{aligned}
$$

If we combine (2), (3), (4) and (5), we obtain the estimate

$$
\begin{aligned}
& \int_{C_{\rho}}|f(t)-f(\omega(t))| \cdot|d t| \\
& \quad \leqq 2 K_{4} R_{2} \sqrt{1-r}+\frac{R_{2}}{1-r}\left[2 \pi K_{3}\left(\frac{\varepsilon}{R_{1}}\right)\right]^{1 / 2}, 0<r<1
\end{aligned}
$$

(d) The whole argument can be repeated with $g(z)$ in place of$f(z)$. In this case we shall have an estimate analogous to (6): 


$$
\begin{aligned}
& \int_{C_{\rho}}\left|g(t)-g\left(\omega_{1}(t)\right)\right| \cdot|d t| \\
& \quad \leqq 2 K_{4} R_{2} \sqrt{1-r}+\frac{R_{2}}{1-r}\left[2 \pi K_{3}\left(\frac{\varepsilon}{R_{1}}\right)\right]^{1 / 2}, 0<r<1 .
\end{aligned}
$$

The function $\omega_{1}(z)$ is the normalized mapping function of $|z|<1$ onto the image of $\Delta$ under the inverse of $g(z)$. Since $f(\omega(z))$ and $g\left(\omega_{1}(z)\right)$ are both normalized mapping functions from $|z|<1$ onto $\Delta$ it follows from the uniqueness that

$$
f(\omega(z)) \equiv g\left(\omega_{1}(z)\right),|z|<1 .
$$

If we combine $(6),\left(6^{\prime}\right),(7)$ and choose $r$ so that $1-r=\left(\varepsilon / R_{1}\right)^{1 / 3}$, the conclusion of the theorem is established.

Throughout the remainder of the paper we shall assume the situation of Theorem 1 with the added hypothesis that $\Gamma_{f}$ and $\Gamma_{g}$ are rectifiable Jordan curves of lengths $L_{f}$ and $L_{g}$. In this case it is well-known that $\bar{D}_{f}$ is the continuous image of $|z| \leqq 1$ and that if $f^{\prime}(z)^{\prime}$ is defined at the boundary by

$$
f^{\prime}\left(e^{i \theta}\right)=\operatorname{Lim}_{z \rightarrow \theta^{i \theta}} \frac{f(z)-f\left(e^{i \theta}\right)}{z-e^{i \theta}},|z| \leqq 1,
$$

then $f^{\prime}\left(e^{i \theta}\right)$ exists almost everywhere, is Lebesgue summable, and

$$
L_{f}=\int_{0}^{2 \pi}\left|f^{\prime}\left(e^{i \theta}\right)\right| d \Theta
$$

3. The following definition ([4], p. 337) and lemma ([4], p. 337) are useful.

Definition $\alpha$. Let $c$ denote a crosscut of $D_{f}$ which does not pass through $w=0$. Let $T$ denote that subregion of $D_{f}$ determined by $c$ which does not contain $w=0$. Let $\lambda$ denote the diameter of $c$ and let $\Lambda$ denote the diameter of $T$. For any $\delta>0$ consider all possible crosscuts $c$ for which $\lambda \leqq \delta$. The crosscut modulus is defined is defined to be

$$
\eta_{f}(\delta)=\sup _{\lambda \geqq \delta} \Lambda
$$

The crosscut modulus is monotonic and has the property:

$$
\eta_{f}(\delta) \rightarrow 0 \text { as } \delta \rightarrow 0
$$

Lemma D. Let $A_{f}$ denote the area of $D_{f}$. Let $z_{0}$ be any point on $|z|=1$ and $k_{s}$ the part of the circle $\left|z-z_{0}\right|=s$ which lies in 
$|z|<1$. Then for every $s, 0<s<1$, there exists a $\sigma, s \leqq \sigma \leqq s^{1 / 2}$ such that the image of $k_{\sigma}$ is a crosscut of length

$$
l_{o} \leqq\left(\frac{2 \pi A_{f}}{\log \frac{1}{s}}\right)^{1 / 2}
$$

We introduce the abbreviation:

$$
\nu_{f}(\delta)=\eta_{f}\left(\left(\frac{2 \pi A_{f}}{\log \frac{1}{\delta}}\right)^{1 / 2}\right), 0<\delta<1 .
$$

An immediate consequence of Lemma $\mathrm{D}$ is

LEMMA 1.

$$
h_{f}(r)=\operatorname{Sup}_{|z|=1}|f(z)-f(r z)| \leqq \nu_{f}(1-r), 0<r<1 .
$$

4. Definition 2. For $m \geqq 2$, let $\left\{w_{1}, w_{2}, w_{3}, \cdots, w_{m}\right\}$ be any set of $m$ distinct points taken in cyclic order on $\Gamma_{f}$ and so distributed that $\Gamma_{f}$ is partitioned into $m$ subarcs of equal length, each subarc having length $L_{f / m}$. Let $l_{\lambda}$ be the length of the perimeter of the cyclically determined polygon, and let $\lambda$, the norm of the partition, be defined by

$$
\lambda=\operatorname{Max}\left[\left|w_{1}-w_{m}\right|,\left|w_{2}-w_{1}\right|,\left|w_{3}-w_{2}\right|, \cdots,\left|w_{m}-w_{m-1}\right|\right] .
$$

The number $l_{\lambda}$ can be written as

$$
l_{\lambda}=\left|w_{1}-w_{m}\right|+\sum_{k=1}^{m-1}\left|w_{k+1}-w_{k}\right| \text {. }
$$

For any $\delta>0$ consider all partitions for which $\lambda \leqq \delta$. Let

$$
U_{f}(\delta)=\operatorname{Inf}_{\lambda \leqq \delta} l_{\lambda}
$$

It is easily shown that $\operatorname{Sup} U_{f}(\delta)=L_{f}$. We define the modulus of rectifiability to be

$$
\zeta_{f}(\delta)=L_{f}-U_{f}(\delta)
$$

The modulus $\zeta_{f}(\delta)$ is monotonic and has the property: $\zeta_{f}(\delta) \rightarrow 0$ as $\delta \rightarrow 0$.

Lemma 2. If $L_{f}(r)$ is the length of the level curve in $D_{f}$ which is the image of $|z|=r$, then

$$
\begin{gathered}
L_{f}-L_{f}(r) \leqq \\
\zeta_{f}(\sqrt{\nu(1-r)})+2 L_{f} \sqrt{\nu_{f}(1-r)} \\
+4 \nu_{f}(1-r), 0<r<1 .
\end{gathered}
$$


Proof. Let the positive integer $m$ be defined by

$$
m=\left[\frac{L_{f}}{\sqrt{\nu_{f}(1-r)}}\right]+2 .
$$

Let $w_{1}, w_{2}, \cdots, w_{m}$ be a set of points in cyclic order $\Gamma_{f}$, so arranged that $\Gamma_{f}$ is partitioned into $m$ equal subarcs, each subarc having length $L_{f} / m$. Clearly the norm of the partition does not exceed $L_{f} / m$ and if $l_{m}$ is the length of the perimeter of the polygon, then

$$
L_{f}-l_{m} \leqq \zeta_{f}\left(\frac{L_{f}}{m}\right) \text {. }
$$

We define the points $z_{k}, \widetilde{w}_{k}$ by $w_{k}=f\left(z_{k}\right), \widetilde{w}_{k}=f\left(r z_{k}\right)$. The set $\widetilde{w}_{k}$ determines a polygon inscribed in the level curve in $D_{f}$ which is the image of $|z|=r$. Comparing corresponding sides of the polygons, we have from Lemma 1 ,

$$
\begin{aligned}
\left|w_{k+1}-w_{k}\right| & \leqq\left|w_{k+1}-\widetilde{w}_{k+1}\right|+\left|\widetilde{w}_{k+1}-\widetilde{w}_{k}\right|+\left|\widetilde{w}_{k}-w_{k}\right| \\
& \leqq h_{f}(r)+\left|\widetilde{w}_{k+1}-\widetilde{w}_{k}\right|+h_{f}(r) \\
& \leqq 2 \nu_{f}(1-r)+\left|\widetilde{w}_{k+1}-\widetilde{w}_{k}\right| .
\end{aligned}
$$

Similarly,

$$
\left|\widetilde{w}_{k+1}-\widetilde{w}_{k}\right| \leqq 2 \nu_{f}(1-r)+\left|w_{k+1}-w_{k}\right| \cdot
$$

Thus, if $l_{m}^{\prime}$ is the length of the perimeter of the level curve polygon,

$$
\left|l_{m}^{\prime}-l_{m}\right| \leqq 2 m \nu_{f}(1-r) \text {. }
$$

Noting that $l_{m}^{\prime} \leqq L_{f}(r)$, we have from (10) and (11)

$$
\begin{aligned}
L_{f}-L_{f}(r) & \leqq L_{f}-l_{m}^{\prime} \leqq L_{f}-l_{m}+\left|l_{m}-l_{m}^{\prime}\right| \cdot \\
& \leqq \zeta_{f}\left(\frac{L_{f}}{m}\right)+2 m \nu_{f}(1-r) .
\end{aligned}
$$

From (9)

$$
\frac{L_{f}}{\sqrt{\nu_{f}(1-r)}} \leqq m \leqq \frac{L_{f}}{\sqrt{\nu_{f}(1-r)}}+2 .
$$

The conclusion follows from (10), (11) and (12).

In the estimate of Lemma 2, it would appear that the first term should dominate the others and this will be so if $\zeta_{f}$ is sufficiently weak. However, it is possible (e.g., if $D_{f}$ is a disk) for the term $2 L_{f} \sqrt{\nu_{f}}$ to be dominant. For purpose of final estimate we introduce the boundary functional

$$
\beta_{f}(\delta)=\zeta_{f}\left(\sqrt{\nu_{f}(\delta)}\right)+2 L_{f} \sqrt{\nu_{f}(\delta)}+4 \nu_{f}(\delta), 0<\delta<1 .
$$


LEMMA 3.

$$
\begin{gathered}
\int_{C_{\rho}}\left|f^{\prime}(t)-f^{\prime}(r t)\right| \cdot|d t| \leqq 2 \sqrt{L_{f} \beta_{f}(1-r)}, \\
0<r<1, \text { for all } \rho, 0 \leqq \rho<1 .
\end{gathered}
$$

Proof. The function $\sqrt{f^{\prime}(z)}$ (i.e., the branch which is positive at the origin) is regular in $|z|<1$. If $\sqrt{f^{\prime}(z)}=\sum_{0}^{\infty} c_{k} z^{k}$, it is well known that $\Sigma\left|c_{k}\right|^{2}$ is convergent and

$$
\begin{aligned}
L_{f} & =\int_{0}^{2 \pi} \sqrt{f^{\prime}\left(e^{i \theta}\right) \sqrt{f^{\prime}\left(e^{i \theta}\right)} d \Theta=2 \pi \Sigma\left|c_{k}\right|^{2},} \\
L_{f}(r) & =\int_{0}^{2 \pi} \sqrt{f^{\prime}\left(r e^{i \theta}\right)} \overline{\sqrt{f^{\prime}\left(r e^{i \theta}\right)}} r d \Theta=2 \pi r \cdot \Sigma\left|c_{k}\right|^{2} r^{2 k}, 0<r<1 .
\end{aligned}
$$

We write

$$
\begin{aligned}
{\left[\int_{C_{\rho}}\right.} & \left.\left|f^{\prime}(t)-f^{\prime}(r t)\right| \cdot|d t|\right]^{2} \\
& \leqq \int_{C_{\rho}}\left|\sqrt{f^{\prime}(t)}-\sqrt{f^{\prime}(r t)}\right|^{2} \cdot|d t| \cdot \int_{C_{\rho}}\left|\sqrt{f^{\prime}(t)}+\sqrt{f^{\prime}(r t)}\right|^{2} \cdot|d t| \\
& =I_{1} \cdot I_{2}, \\
I_{1} & =2 \pi \rho \cdot \Sigma\left|c_{k}\right|^{2} \rho^{2 k}\left(1-2 r^{k}+r^{2 k}\right) \leqq 2 \pi \Sigma\left|c_{k}\right|^{2}\left(1-r^{2 k}\right) \\
& =L_{f}-L_{f}(r) \cdot \frac{1}{r} \leqq L_{f}-L_{f}(r), \\
I_{2} & =2 \pi \rho \Sigma\left|c_{k}\right|^{2} \rho^{2 k}\left(1+2 r^{k}+r^{2 k}\right) \leqq 2 \pi \Sigma\left(\left|c_{k}\right|^{2} \cdot 4\right)=4 L_{f} .
\end{aligned}
$$

From these inequalities and Lemma 2, the conclusion is apparent.

\section{Final estimates. We assert:}

THEOREM 2. If $\Gamma_{f}$ and $\Gamma_{g}$ are rectifiable Jordan curves of lengths $L_{f}$ and $L_{g}$, if $0<\varepsilon / R_{1}<1$, then

$$
\begin{gathered}
I\left(f^{\prime}, g^{\prime} ; \rho\right) \leqq 2\left[\sqrt{L_{f}}+\sqrt{L_{g}}+M / R_{1}^{1 / 2}\right] \sqrt{\beta_{f}(\sigma)}+2 \sqrt{L_{g} \mu}, \\
\text { uniformly for all } \rho, 0 \leqq \rho<1,
\end{gathered}
$$

where $\sigma=\left(\varepsilon / R_{1}\right)^{1 / 24}, \mu=\left|L_{f}-L_{g}\right|, M=\operatorname{Max}\left[K_{1} R_{2}, 2 \sqrt{L_{g} K_{1} R_{2}}\right]$.

Proof. Write

$$
\begin{aligned}
I\left(f^{\prime}, g^{\prime} ; \rho\right) \leqq & \int_{C_{\rho}}\left|f^{\prime}(t)-f^{\prime}(r t)\right| \cdot|d t|+\int_{C_{\rho}}\left|f^{\prime}(r t)-g^{\prime}(r t)\right| \cdot|d t| \\
& +\int_{C_{\rho}}\left|g^{\prime}(r t)-g^{\prime}(t)\right| \cdot|d t|=I_{1}+I_{2}+I_{3} .
\end{aligned}
$$

Choose $1-r=\sigma$, from Lemma 3 , 


$$
I_{1} \leqq 2 \sqrt{L_{f} \beta_{f}(\sigma)} \text {. }
$$

Let $0<\rho<\alpha<1$, then, from Theorem 1

$$
\begin{aligned}
I_{2} & \leqq \int_{C_{\rho}}\left[\frac{1}{2 \pi} \int_{C_{\alpha}}\left|\frac{f(\gamma)-g(\gamma)}{(\gamma-r t)^{2}}\right| \cdot|d \gamma|\right] \cdot|d t| \leqq \frac{K_{1} R_{2}\left(\varepsilon / R_{1}\right)^{1 / 8}}{(1-r)^{2}} \\
& =K_{1} R_{2} \sigma^{2} \leqq K_{1} R_{2} \sigma .
\end{aligned}
$$

From the proof of Lemma 3 (with $g$ in place of $f$ )

$$
I_{3} \leqq 2 \sqrt{L_{g}}\left(L_{g}-L_{g}(r)\right)^{1 / 2},
$$

and

$$
\begin{aligned}
L_{g}-L_{g}(r) & \leqq\left|L_{g}-L_{f}\right|+L_{f}-L_{f}(r)+\left|L_{f}(r)-L_{g}(r)\right| \\
& =\mu+A+B .
\end{aligned}
$$

From Lemma $2, A \leqq \beta_{f}(\sigma)$, and

$$
\begin{aligned}
B & =\left|\int_{C_{\rho}}\left[\left|f^{\prime}(r t)\right|-\left|g^{\prime}(r t)\right|\right]\right| d t\left|\leqq \int_{C_{\rho}}\right| f^{\prime}(r t)-g^{\prime}(r t)|\cdot| d t|| \\
& =I_{2} \leqq K_{1} R_{2} \sigma^{2} .
\end{aligned}
$$

Thus,

$$
I_{3} \leqq 2 \sqrt{L_{g}}(\mu+A+B)^{1 / 2} \leqq 2 \sqrt{L_{g}}\left(\mu^{1 / 2}+A^{1 / 2}+B^{1 / 2}\right) .
$$

Combining estimates we have

$$
\begin{aligned}
I\left(f^{\prime}, g^{\prime} ; \rho\right) \leqq & 2\left(\sqrt{L_{f}}+\sqrt{L_{g}}\right) \sqrt{\beta_{f}(\sigma)} \\
& +2 \sqrt{L_{g} \mu}+\left(2 \sqrt{L_{g} K_{1} R_{2}}+K_{1} R_{2}\right) \sigma .
\end{aligned}
$$

From (8) and (13) and the definition of $\eta_{f}$,

$$
\begin{aligned}
\sqrt{\beta_{f}(\sigma)} & \geqq 2\left(\nu_{f}(\sigma)\right)^{1 / 2} \geqq \eta_{f}\left(\left(\frac{2 \pi A_{f}}{\log \frac{1}{\sigma}}\right)^{1 / 2}\right)^{1 / 2} \geqq\left(\frac{2 \pi A_{f}}{\log \frac{1}{\sigma}}\right)^{1 / 4} \\
& \geqq\left(\left(2 \pi^{2} R_{1}^{2}\right)^{1 / 4} \cdot \sigma\right) \geqq R_{1}^{1 / 2} \sigma .
\end{aligned}
$$

Hence

$$
\left(2 \sqrt{L_{g} K_{1} R_{2}}+K_{1} R_{2}\right) \sigma \leqq \frac{2 M \sqrt{\beta_{f}(\sigma)}}{R_{1}^{1 / 2}}
$$

the conclusion follows from (14) and (15).

LEMMA 4. If $\mu=\left|L_{f}-L_{g}\right|$ and if

$$
I^{*}=\operatorname{Sup}_{\rho} I\left(f^{\prime}, g^{\prime} ; \rho\right), 0 \leqq \rho<1 \text {, then } \mu \leqq I^{*} \text {. }
$$


Proof. We have

$$
\begin{aligned}
& \left|L_{f}(\rho)-L_{g}(\rho)\right|=\left|\int_{C_{\rho}}\right| f^{\prime}(t)|\cdot| d t\left|-\int_{C_{\rho}}\right| g^{\prime}(t)|\cdot| d t|| \\
& \leqq\left|\int_{C_{\rho}}\right| f^{\prime}(t)-g^{\prime}(t)|\cdot| d t \mid=I\left(f^{\prime}, g^{\prime} ; \rho\right) \leqq I^{*}
\end{aligned}
$$

Let $\rho \rightarrow 1$ on the left and the lemma is proved.

LEMMA 5.

$$
\left|f\left(e^{i \theta}\right)-g\left(e^{i \theta}\right)\right| \leqq I^{*}
$$

Proof. The Fejer-Riesz inequality asserts that

$$
\begin{aligned}
A=\int_{-1}^{1}|H(x)|^{p} d x \leqq \frac{1}{2} \int_{0}^{2 \pi}\left|H\left(e^{i \alpha}\right)\right|^{p} d \alpha & =B, \\
p & >0 \text { and } x \text { is real } .
\end{aligned}
$$

Here $H(z)$ which is regular in $|z|<1$ belongs to the Hardy class $H^{p}$ in $|z| \leqq 1$. Let $p=1$ and we make the choice $H(z)=\rho e^{i \theta}\left(f^{\prime}\left(z \rho e^{i \theta}\right)-\right.$ $\left.g^{\prime}\left(z \rho e^{i \theta}\right)\right)$. Noting that $A \geqq\left|\int_{0}^{1} H(x) d x\right|$, that $2 B=I\left(f^{\prime}, g^{\prime} ; \rho\right) \leqq I^{*}$, we let $\rho \rightarrow 1$ and we get the conclusion of the lemma.

We are now able to state our convergence theorem as

THEOREM 3. If the $f$ boundary is held fixed and the $g$ boundary is allowed to vary, a necessary and sufficient condition that $I\left(f^{\prime}, g^{\prime} ; \rho\right) \rightarrow$ 0 uniformly for all $\rho, 0 \leqq \rho<1$, is that $\mu+\sigma \rightarrow 0$.

Proof. We get the sufficiency from Theorem 2. From Lemma 4 we see that $I^{*} \rightarrow 0$ implies that $\mu \rightarrow 0$ which is one part of the necessity. From Lemma 5, we see that if $I^{*}$ is arbitrarily small the boundary point $f\left(e^{i \theta}\right)$ will be arbitrarily close to the $g$ boundary and vice versa. So we have $I^{*} \rightarrow 0$ implies $\varepsilon \rightarrow 0$ implies that inf $R_{1}>0$ so that $I^{*} \rightarrow 0$ implies that $\sigma \rightarrow 0$. This completes the proof of Theorem 3.

Without estimate, S. E. Warschawski [2] established a result that is similar to Theorem 3 .

\section{BIBLIOGRAPHY}

1. J. E. Littlewood, Lectures on the theory of functions, Oxford University Press, London, England, 1944.

2. S. E. Warschawski, Über einige Konvergensätze aus der Theorie der konformen Abbildung, Nachrichten von der Gesellschaft der Wissenschaften, Göttingen, 1930.

3. — On conformal mapping of nearly circular regions, Proc. Amer. Math. 
Soc. 1 (1950), 562-574.

4. - On the degree of variation in conformal mapping of variable regions, Trans. Amer. Math. Soc. 69 (1950), 335-356.

Received February 12, 1968. This paper gives the main results of a thesis which originated in 1950 at the University of Minnesota under the direction of Professor S. E. Warschawski, to whom the author is grateful for valuable assistance. 



\section{PACIFIC JOURNAL OF MATHEMATICS}

EDITORS

H. SAMELSON

Stanford University

Stanford, California 94305

RichaRd PIERCE

University of Washington

Seattle, Washington 98105
J. DUGUNDJI

Department of Mathematics

University of Southern California

Los Angeles, California 90007

RICHARD ARENS

University of California

Los Angeles, California 90024

\section{ASSOCIATE EDITORS}

E. F. BECKENBACH

B. H. NeumanN

F. WOLF

K. YOSHIDA

\section{SUPPORTING INSTITUTIONS}

UNIVERSITY OF BRITISH COLUMBIA

CALIFORNIA INSTITUTE OF TECHNOLOGY

UNIVERSITY OF CALIFORNIA

MONTANA STATE UNIVERSITY

UNIVERSITY OF NEVADA

NEW MEXICO STATE UNIVERSITY

OREGON STATE UNIVERSITY

UNIVERSITY OF OREGON

OSAKA UNIVERSITY

UNIVERSITY OF SOUTHERN CALIFORNIA
STANFORD UNIVERSITY

UNIVERSITY OF TOKYO

UNIVERSITY OF UTAH

WASHINGTON STATE UNIVERSITY

UNIVERSITY OF WASHINGTON

AMERICAN MATHEMATICAL SOCIETY CHEVRON RESEARCH CORPORATION TRW SYSTEMS

NAVAL WEAPONS CENTER 


\section{Pacific Journal of Mathematics}

May, 1970

Johan Aarnes, Edward George Effros and Ole A. Nielsen, Locally compact

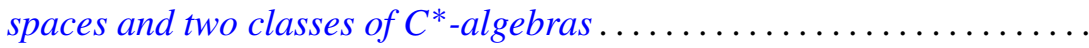

Allan C. Cochran, R. Keown and C. R. Williams, On a class of topological

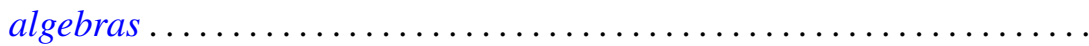

John Dauns, Integral domains that are not embeddable in division rings ....

Robert Jay Daverman, On the number of nonpiercing points in certain

crumpled cubes.....................................

Bryce L. Elkins, Characterization of separable ideals ................

Zbigniew Fiedorowicz, A comparison of two naturally arising uniformities

on a class of pseudo-PM spaces ...........................

Henry Charles Finlayson, Approximation of Wiener integrals of functionals

continuous in the uniform topology ........................

Theodore William Gamelin, Localization of the corona problem ...........

Alfred Gray and Paul Stephen Green, Sphere transitive structures and the

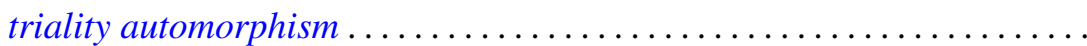

Charles Lemuel Hagopian, On generalized forms of aposyndesis ..........

J. Jakubík, On subgroups of a pseudo lattice ordered group ...............

Cornelius W. Onneweer, On uniform convergence for Walsh-Fourier

series..................................

Stanley Joel Osher, On certain Toeplitz operators in two variables ...

Washek (Vaclav) Frantisek Pfeffer and John Benson Wilbur, On the

measurability of Perron integrable functions............

Frank J. Polansky, On the conformal mapping of variable regions...

Kouei Sekigawa and Shûkichi Tanno, Sufficient conditions for a Riemannian manifold to be locally symmetric ...................

James Wilson Stepp, Locally compact Clifford semigroups ....

Ernest Lester Stitzinger, Frattini subalgebras of a class of solvable Lie

algebras ................................

George Szeto, The group character and split group algebras...

Mark Lawrence Teply, Homological dimension and splitting torsion

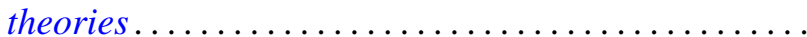

David Bertram Wales, Finite linear groups of degree seven. II ...

Robert Breckenridge Warfield, Jr., An isomorphic refinement theorem for

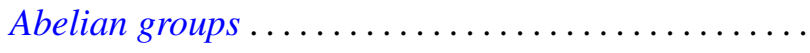

James Edward West, The ambient homeomorphy of an incomplete subspace

of infinite-dimensional Hilbert spaces................

Peter Wilker, Adjoint product and hom functors in general topology ...

Daniel Eliot Wulbert, A note on the characterization of conditional 